\title{
Uso de allium cepa test como indicador de eficacia para el tratamiento de efluentes
}

\author{
Estela Mónica López Sardi, 'Beatriz Noemí García, i Victoria Larroudé, iii \\ Ricardo Picicelli, ${ }^{i v}$ Carla Yanina Reynosov y Erica Ramírez Martínez ${ }^{\mathrm{vi}}$
}

\section{Resumen}

Cuando los vertidos líquidos de una industria se descargan a un conducto pluvial o a la red cloacal general, la probabilidad de que estos efluentes terminen en un curso de agua superficial o subterránea es muy alta. La disminución o eliminación total de la biotoxicidad de estos efluentes antes de su descarte es de vital importancia para prevenir impactos negativos sobre la biodiversidad del ecosistema que recibe los vertidos.

El uso del test de Allium cepa como bioindicador en ensayos de biotoxicidad y genotoxicidad está reconocido a nivel mundial. En el presente trabajo proponemos utilizar el test como un indicador para evaluar la eficacia de los tratamientos que las

Cuando los vertidos líquidos de una industria se descargan a un conducto pluvial o a la red cloacal general, la probabilidad de que estos efluentes terminen en un curso de agua superficial o subterránea es muy alta. La disminución o eliminación total de la biotoxicidad de estos efluentes antes de su descarte es de vital importancia para prevenir impactos negativos sobre la biodiversidad del ecosistema que recibe los vertidos.

El uso del test de Allium cepa como bioindicador en ensayos de biotoxicidad y genotoxicidad está reconocido a nivel mundial. En el presente trabajo proponemos utilizar el test como un indicador para evaluar la eficacia de los tratamientos que las industrias realizan sobre sus efluentes líquidos antes del vertido a la red cloacal general.

Profesora Titular de Química General e Investigadora de la Facultad de Ingeniería, Universidad de Palermo.

i Profesora Titular de Química General II e Investigadora de la Facultad del Ejército. Universidad de la Defensa Nacional.

iii Jefe de Trabajos Prácticos de Química General e Investigadora de la Facultad de Ingeniería, Universidad de Palermo.

iv Profesor de Física II e Investigador de la Facultad del Ejército. Universidad de la Defensa Nacional. Profesor Física A y B de la Facultad de Ingeniería, Universidad de Palermo.

$\checkmark$ Jefe de Trabajos Prácticos de Química General I e Investigadora de la Facultad del Ejército. Universidad de la Defensa Nacional.

vi Investigadora de la Facultad del Ejército. Universidad de la Defensa Nacional. 
Palabras clave: vertidos industriales, eficacia del tratamiento de efluentes, Allium cepa test.

\section{Abstract}

When the industry's liquid effluents are discharged to a storm duct or to the general sewage network, the probability that these effluents end up in a course of surface water or groundwater is very high. The reduction or total elimination of the biotoxicity of these effluents prior to disposal is vital to prevent negative impacts on biodiversity of the ecosystem that receives the discharges.

The use of Allium cepa test as a biomarker in genotoxicity and biotoxicity assays is recognized worldwide. In this paper we propose the use of the test as an indicator to analyze the effectiveness of the treatments that industries performed on its liquid effluents before discharge them to the general sewer system.

Key words: industrial liquid waste, effluent treatment effectiveness, Allium cepa test. 


\section{Introducción}

El test de Allium cepa es de utilidad para conducir investigaciones sobre aguas, ya sean de consumo humano, de depósitos municipales, aguas superficiales o subterráneas, efluentes cloacales u otras. El Programa Internacional de Bioensayos Vegetales, La Real Academia Sueca de las Ciencias y el GENE - TOX PROGRAM, entre otros, alientan su uso como bioindicador para ensayos de biotoxicidad y genotoxicidad. (Firbas, 2011).

Cuando un bulbo de la variedad de cebolla Allium sp. se rehidrata se produce una estimulación del crecimiento de las células, lo cual permite la elongación de las raíces de la planta. Sin embargo, cuando la hidratación se lleva a cabo en presencia de sustancias tóxicas, la división celular de los meristemos radiculares puede inhibirse, ya sea retardando el proceso de mitosis o destruyendo las células. Este tipo de alteraciones generalmente impide el crecimiento normal de la raíz, y por tanto su elongación. El efecto puede determinarse en forma indirecta, mediante la comparación de la elongación de las raíces de los bulbos expuestos al compuesto tóxico contra la de bulbos no expuestos. La cuantificación del efecto se realiza estableciendo el porcentaje de inhibición del crecimiento $(\% \mathrm{IC})$ de las raíces expuestas al tóxico respecto a la longitud promedio de las raíces del control. (Díaz Báez et al.; 2004).

La importancia del Allium cepa test radica en que es un excelente modelo de ensayo in vivo, donde es posible evaluar el daño producido por una sustancia o solución de interés sobre el crecimiento de las raíces y sobre el ADN vegetal. Es ventajoso el uso del Allium cepa test por emplearse como indicador una planta vascular, que permite configurar un modelo excelente para realizar ensayos sobre sustancias contaminantes, mutagénicas o cancerígenas cuyos datos pueden ser extrapolados a toda la biodiversidad de plantas y animales. (Bosio Tedesco et al., 2012).

\section{Objetivos de la experiencia}

Cuando los vertidos líquidos de una industria se descargan a un conducto pluvial o a la red cloacal general, la probabilidad de que estos efluentes terminen en un curso de agua superficial o subterránea es muy alta. La disminución o eliminación total de la biotoxicidad de estos efluentes antes de su descarte es de vital importancia para prevenir impactos negativos sobre la biodiversidad del ecosistema que recibe los vertidos. La experiencia se llevó a cabo con el objetivo de evaluar si es posible utilizar el Allium cepa test como herramienta para determinar la eficacia de los tratamientos realizados sobre los efluentes industriales con el fin de disminuir su biotoxicidad. 


\section{Materiales y métodos}

Para llevar adelante la experiencia se tomaron muestras de efluentes en dos industrias del rubro laboratorio farmacéutico veterinario. En ambos casos se tomaron dos muestras de efluentes: antes de ser sometidos al tratamiento y después de ser sometidos al tratamiento. Se realizó el Allium cepa test sobre las muestras de efluentes concentrados tratados y sin tratar y sobre sus sus diluciones acuosas 1:2 y 1:10. Como medio de control se utilizó agua del grifo, cuya calidad se considera no tóxica y totalmente inofensiva hacia el ambiente, en caso de ser descartada en el sistema cloacal.

Los ensayos y el control se llevaron a cabo por duplicado. Como parámetro de evaluación se utilizó la elongación de las raíces desarrolladas durante 96 horas a partir del momento de la puesta en contacto de los bulbos con los distintos medios de crecimiento. Durante el período de crecimiento de las raíces el laboratorio estuvo iluminado con luz natural indirecta y la temperatura se mantuvo en el rango de los $20-25^{\circ} \mathrm{C}$.

Se sembraron bulbos de $5 \mathrm{~cm}$ de diámetro de la familia Amaryllidaceae como se observa en la Imagen 1.

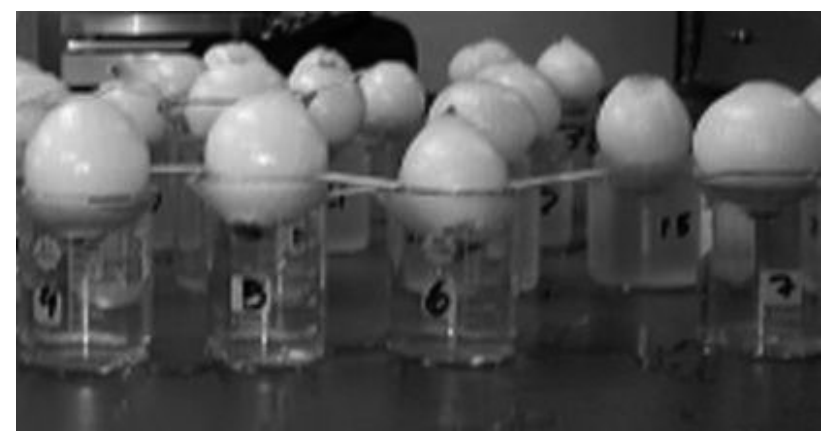

Imagen 1. Allium cepa test: Siembra de bulbos de la familia Amaryllidaceae.

Los discos caulinares de estos bulbos, antes de la siembra, deben estar libres de raíces desarrolladas. En caso de existir alguna, se la debe cortar al nivel del disco. El sistema radicular de los bulbos está formado por numerosas raicillas de longitud variable, fasciculadas, blanquecinas y poco profundas, que se desarrollarán a partir del disco caulinar, en contacto con los distintos medios de cultivo, como se puede ver en la Imagen 2. (Rothman, 2008). Luego de transcurridas las 96 horas, las raicillas desarrolladas se cortaron a nivel del disco. Se midió la longitud de cada una de ellas utilizando reglas metálicas de precisión con resolución $0,5 \mathrm{~mm}$. 


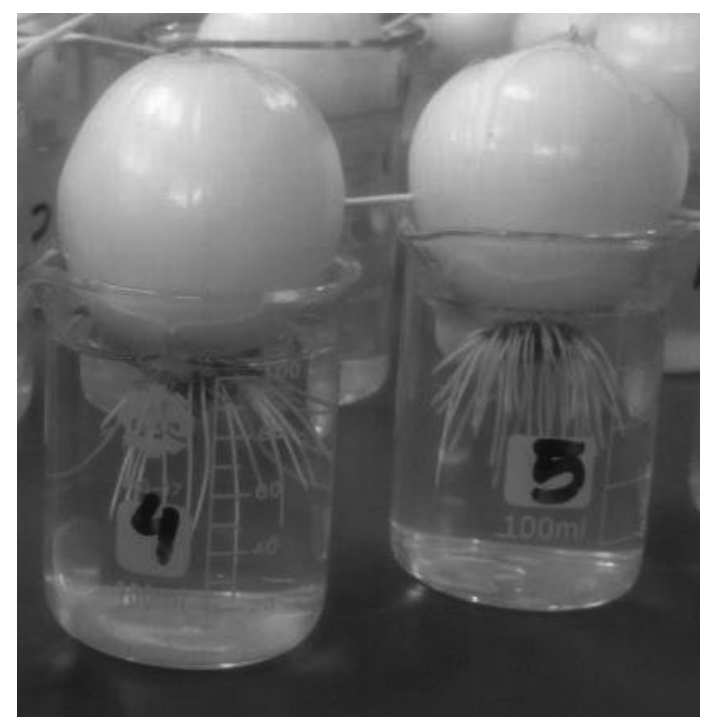

Imagen 2. Allium cepa test: elongación de raíces.

Los establecimientos en los que se tomaron las muestras serán denominados en adelante Establecimiento 1 (E1) y Establecimiento 2 (E2), por razones de confidencialidad. A continuación se detallan algunas características de cada uno de los establecimientos.

E1: Laboratorio de elaboración de productos biológicos, vacunas, productos terapéuticos y farmacológicos para pequeños y grandes animales. Localizado en la zona Oeste de la Ciudad de Buenos Aires (barrio de Mataderos). Los residuos biológicos se tercerizan para su tratamiento, derivándolos a una planta de tratamiento especializado. Los efluentes que se vuelcan a la red cloacal general luego de su tratamiento, son los que provienen de limpieza del establecimiento, de maquinarias y otros usos generales. Estos efluentes son volcados en un tanque de depósito especial, donde son aireados y tratados con hipoclorito de sodio. Luego se vuelcan a la red general. Se tomaron muestras de los efluentes antes y después de pasar por el tanque de tratamiento.

E2: Laboratorio de elaboración de vacunas, reactivos y antígenos de diagnóstico veterinario. Ubicado en la zona de Pilar. Los efluentes pasan por sedimentadores primarios y secundarios con puente barredor para el descarte de lodos. Se tomaron muestras de los efluentes antes del tratamiento y a la salida del puente. Al igual que en el caso anterior, los residuos biológicos se tercerizan para su tratamiento. 


\section{Resultados}

La Tabla 1 contiene la descripción de muestras analizadas en referencia al establecimiento $\boldsymbol{E} 1$.

\begin{tabular}{|l|}
\hline \multicolumn{1}{|c|}{ MUESTRAS $\boldsymbol{E 1}$} \\
\hline 1.- Control de agua potable del grifo \\
2.- Efluentes sin tratar concentrados \\
3.- Efluentes sin tratar dilución $1: 2$ \\
4.- Efluentes sin tratar dilución $1: 10$ \\
5.- Efluentes tratados concentrados \\
6.- Efluentes tratados dilución $1: 2$ \\
7.- Efluentes tratados dilución $1: 10$ \\
\hline
\end{tabular}

Tabla 1. Descripción de muestras de E1.

Los resultados del test aplicado a las muestras de $\boldsymbol{E} 1$, se resumen en la Tabla 2.

\begin{tabular}{|l|l|l|l|l|l|l|l|}
\hline MUESTRA & 1 & 2 & 3 & 4 & 5 & 6 & 7 \\
\hline MÍN & 1,2 & 1 & 1,6 & 1,7 & 1,5 & 1 & 1 \\
\hline MÁX & 10 & 6,3 & 9,8 & 8,6 & 2,1 & 1 & 4,9 \\
\hline RANGO & 8,8 & 5,3 & 8,2 & 6,9 & 0,6 & 0 & 3,9 \\
\hline MEDIA & 6,49574468 & 3,17647059 & 5,71325301 & 5,915625 & 1,85714286 & 1 & 2,78627451 \\
\hline \%IC & 0 & 51,1 & 12,05 & 8,93 & 71,41 & 84,61 & 57,11 \\
\hline MODO & 6 & 2,5 & 4,4 & 6,5 & 1,9 & 1 & 3,3 \\
\hline MEDIANA & 6,3 & 3 & 5,8 & 6,5 & 1,9 & 1 & 2,9 \\
\hline DESVÍO M & 2,7016064 & 1,37529462 & 1,90056289 & 1,72460549 & 0,22253946 & 0 & 0,90443786 \\
\hline $\begin{array}{l}\text { DESVÍO } \\
\text { POB }\end{array}$ & 2,67271139 & 1,36174459 & 1,88907902 & 1,69744466 & 0,2060315 & 0 & 0,895526925 \\
\hline
\end{tabular}

Tabla 2. Resultados obtenidos del Allium cepa test sobre las muestras de E1.

La Tabla 3 contiene la descripción de muestras analizadas en referencia al establecimiento $\boldsymbol{E} 2$.

\begin{tabular}{|l|}
\hline \multicolumn{1}{|c|}{ MUESTRAS E2 } \\
\hline 1.- Control de agua potable del grifo \\
2.- Efluentes sin tratar concentrados \\
3.- Efluentes sin tratar dilución $1: 2$ \\
4.- Efluentes sin tratar dilución $1: 10$ \\
5.- Efluentes tratados concentrados \\
6.- Efluentes tratados dilución $1: 2$ \\
7.- Efluentes tratados dilución $1: 10$ \\
\hline
\end{tabular}

Tabla 3. Descripción de muestras de E2. 
Los resultados del test aplicado a las muestras de $\boldsymbol{E} \mathbf{2}$, se resumen en la Tabla 4.

\begin{tabular}{|l|l|l|l|l|l|l|l|}
\hline MUESTRA & 1 & 2 & 3 & 4 & 5 & 6 & 7 \\
\hline MÍN & 1,2 & 0,5 & 1 & 1 & 0,5 & 0,5 & 1,5 \\
\hline MÁX & 10 & 6 & 5,8 & 6,2 & 9,3 & 7,5 & 8,1 \\
\hline RANGO & 8,8 & 5,5 & 4,8 & 5,2 & & 8,8 & 6,6 \\
\hline MEDIA & 6,49574468 & 3,58 & 3,49111111 & 2,98688525 & 4,07272727 & 4,57816092 & 4,88269231 \\
\hline \%IC & 0 & 44,89 & 46,25 & 54,02 & 37,3 & 29,52 & 24,83 \\
\hline MODO & 6 & 1,2 & 3,5 & 1 & - & 1 & 6,2 \\
\hline MEDIANA & 6,3 & 3,8 & 3,9 & 3 & 4,1 & 5,3 & 5,4 \\
\hline DESVÍO M & 2,7016064 & 1,75102087 & 1,44030019 & 1,39707736 & 2,59893418 & 2,08518725 & 1,92371385 \\
\hline $\begin{array}{l}\text { DESVÍO } \\
\text { POB }\end{array}$ & 2,67271139 & 1,73502947 & 1,42420695 & 1,38557858 & 2,53918058 & 2,07316878 & 1,9051268 \\
\hline
\end{tabular}

Tabla 4. Resultados obtenidos del Allium cepa test sobre las muestras de E2.

\section{Análisis de resultados}

\section{A. Establecimiento E1}

De acuerdo con los resultados obtenidos para el establecimiento E1 podemos determinar que el tratamiento aplicado a los efluentes ha demostrado un efecto contrario al esperado. Como respuesta al tratamiento se observa un incremento de la biotoxicidad, dada la menor elongación de las raíces desarrolladas en los efluentes tratados y/o sus diluciones respecto de la elongación observada por las raíces desarrolladas en las muestras de efluentes sin tratar y sus diluciones. Este incremento de la biotoxicidad puede atribuirse al agregado del hipoclorito de sodio durante el tratamiento. Al analizar los \% IC (\% de Inhibición del Crecimiento) y demás resultados del test (Tabla 2), observamos que en el caso de esta industria, la dilución al medio con agua del grifo de los efluentes resultaría ser más efectiva para disminuir la biotoxicidad de los vertidos que el tratamiento aplicado.

\section{B. Establecimiento E2}

Los resultados de los ensayos de Allium Cepa test llevados adelante en los efluentes tratados y sin tratar de este establecimiento muestran que el tratamiento aplicado tiene la capacidad de disminuir la biotoxicidad de sus vertidos, si bien no la elimina completamente. Los efluentes tratados concentrados siguen presentando un nivel de \%IC importante, cuya disminución se favorece al complementar el 
tratamiento con la dilución. Mientras que en los efluentes sin tratar del E2 la dilución parece tener un efecto inhibidor del crecimiento cada vez mayor, la dilución de los efluentes tratados revierte esa tendencia, mostrando que a mayor dilución del efluente tratado menor \%IC.

\section{Conclusión}

Los resultados obtenidos a partir de la aplicación del test de Allium cepa nos ha permitido evaluar la eficacia para disminuir la biotoxicidad que tuvieron los tratamientos aplicados a los efluentes de estos dos establecimientos.

Nuestra conclusión es que es posible utilizar el test como indicador de eficacia en el tratamiento de vertidos líquidos industriales.

En el caso de un establecimiento que analiza la posibilidad de elegir entre una modalidad de tratamiento $u$ otra, este test puede aportar valiosa información sobre la efectividad de disminución de la biotoxicidad de las alternativas propuestas.

La realización de una correcta evaluación de la efectividad de los procesos aplicados permitirá mejorar el diseño del tratamiento, muchas veces con ahorro de recursos económicos y optimización de los resultados ambientales.

\section{Agradecimientos}

Los autores queremos agradecer especialmente a los alumnos de la Facultad de Ingeniería de la Universidad de Palermo que tan eficientemente colaboraron en la realización de los aspectos experimentales del trabajo: Alexis Beltrán, Sofía Carneglia, Mauricio de la Cruz y Nicolás Díaz.

\section{Fuentes consultadas}

Firbas, P. (2011), Test Report for Flaska, consultado el 27 de agosto de 2015 en http://www.flaska.si/files/original/756.pdf

Díaz Báez, M. C.; Ronco, A. y Pica granados, Y., (2004), Ensayos toxicológicos y métodos de evaluación de calidad de aguas. Estandarización, intercalibración, resultados y aplicaciones. G. Castillo (Ed.). IDRC, IMTA, Canadá.

Solange Bosio Tedesco and Haywood Dail Laughinghouse IV (2012). Cebolla Allium cepa, Environmental Contamination, Dr. Jatin Srivastava (Ed.), ISBN: 978-953-51-0120-8, InTech, Consultado el 2 de marzo de 2016 en: http:// 
www.intechopen.com/books/environmental-contamination/bioindicator-ofgenotoxicitythe-allium-cepa-test

Rothman, S. y Dondo G., (2008), Cebolla Allium cepa, Cátedra de Horticultura, Departamento producción vegetal, Facultad de ciencias agropecuarias, Universidad nacional de Entre Ríos. Consultado el 20 de abril de 2016 en: http://web.archive.org/web/20131105022125/http://www.fca.uner.edu.ar/ academicas/deptos/catedras/horticultura/cebolla.pdf 
